\title{
Virtual Space Level Shifting and Correlation Energies
}

\author{
JÉRÔME REY, ANDREAS SAVIN \\ Laboratoire de Chimie Théorique (CNRS), Université P. et M. Curie, 4, place Jussieu, \\ F-75252 Paris, France
}

Received 5 June 1997; revised 23 September 1997; accepted 30 September 1997

\begin{abstract}
Adding a nonlocal operator to the true Hamiltonian is used to define an adiabatic coupling between a noninteracting (e.g., Kohn-Sham) reference system and the real one. By using the Hellmann-Feynman theorem, it is shown that when the operator added is shifting upward the virtual (noninteracting) levels the correlation energy is related to the number of electrons displaced into the virtual levels. To construct approximations, calculations were performed for the uniform electron gas. The expectation that atomic systems would behave locally like a uniform electron gas with the unoccupied levels shifted up by a constant close to the atomic excitation energies is not confirmed by exploratory calculations on atoms. Some perturbation theory expressions are also given and suggest an approach to self-interaction free-correlation energy functionals. (c) 1998 John Wiley \& Sons, Inc. Int J Quant Chem 69: 581-590, 1998
\end{abstract}

\section{Introduction}

$\mathbf{0}$ ne of the techniques used to obtain more insight into density functional theory (DFT) and a supplementary flexibility of the functionals themselves is the modification of the Hamiltonian. One common example is the modification of the electron-electron interaction operator either by a constant, (see, e.g., $[1,2]$ for the theoretical aspect or $[3,4]$ for its applications) or by splitting it into a short- and a long-range part, (see, e.g., [5]). Alternatively, one-electron nonlocal operators can be used [6]. Such approaches are promising alterna-

Correspondence to: A. Savin. tives to the widely used Kohn-Sham schemes [7]. As introducing supplementary one-body operators seems to be a good compromise between the wish of supplementary flexibility in density functional schemes and computational flexibility, this article will discuss a very simple one, which shifts up all unoccupied levels of some effective one-electron Hamiltonian by an arbitrary constant.* A motivation for this approach is a question which is sometimes raised in connection with the local density approximation (which transfers the correlation energy per particle from the uniform electron gas): Would it be better to transfer the correlation energy per particle from a uniform electron gas with

* A local one-body operator will, of course, not modify the Kohn-Sham scheme. 
a gap? A physical argument in favor of such a change is the significant difference in the spectrum in a system such as an atom, a molecule, or an insulator, on the one hand, and in the uniform electron gas, on the other hand, the most striking feature being the absence of a gap for the latter. This reminds one, of course, of Callaway's approach for describing insulators [8]. We would like to mention that some of its drawbacks are not present in Penn's model [9], which has been already used as a reference for approximations $[10,11]$. For the sake of simplicity, however, we will stick in the present article to the rigid shift of the energy levels by a constant.

The article is structured as follows: In the first part of the article, we present the level-shifting alternative to the usual (electron-electron interaction switch-on $[1,2])$ adiabatic coupling theorm. The second part contains some aspects of perturbation theory in the present context. Later, some policies of constructing approximations will be shown. For these, the correlation energies of a uniform electron gas with a gap will be given. The last part shows the effect of simple approximations when applied to simple systems like the helium and beryllium atoms.

\section{Adiabatic Coupling Formula}

Let us consider a model $N$-body Hamiltonian $\hat{H}^{G}$ derived from the true one $(\hat{H})$ by adding a given one-body nonlocal operator, $\hat{o}$, multiplied by a positive number $G$ which we will choose to vary. The expectation value is

$$
\left\langle\hat{H}^{G}\right\rangle=\langle\hat{H}\rangle+G \int o\left(r, r^{\prime}\right) \gamma\left(r^{\prime}, r\right) d^{3} r d^{3} r^{\prime},
$$

where $\gamma\left(r^{\prime}, r\right)$ is the reduced one-body density matrix. With the minimizing wave function, one obtains the ground-state energy of the model system, $E^{G}=\left\langle\hat{H}^{G}\right\rangle$. For each $G$, the HellmannFeynman theorem can be applied, yielding

$$
\partial E^{G} / \partial G=\int o\left(r, r^{\prime}\right) \gamma^{G}\left(r^{\prime}, r\right) d^{3} r d^{3} r^{\prime},
$$

where $\gamma^{G}\left(r^{\prime}, r\right)$ is the reduced one-body density matrix obtained from the wave function minimizing $\left\langle\hat{H}^{G}\right\rangle$. $^{\dagger}$ This equation can also be written in

\footnotetext{
${ }^{\dagger}$ See a formally similar approach in [12].
}

integral form:

$$
\begin{aligned}
E^{G_{1}}-E^{G_{2}} & =\int_{G_{2}}^{G_{1}} \partial E^{G} / \partial G d G \\
& =\int_{G_{2}}^{G_{1}} d G \int o\left(r, r^{\prime}\right) \gamma^{G}\left(r^{\prime}, r\right) d^{3} r d^{3} r^{\prime} .
\end{aligned}
$$

Up to now, the operator $\hat{o}$ is general. In this article, however, a simple form will be chosen for $\hat{o}$ :

$$
o_{s}\left(r, r^{\prime}\right)=\sum_{i}^{\text {virtual }} \varphi_{i}(r) \varphi_{i}^{*}\left(r^{\prime}\right),
$$

which is a projection operator onto some orbital space. The orbitals $\varphi_{i}$ can be thought of being the eigenfunctions of some one-body Hamiltonian, e.g., the Kohn-Sham or Hartree-Fock Hamiltonian. We have restricted ourselves again, by limiting the sum to being performed over those not occupied in the ground state of the Hamiltonian. By using the completeness relationship,

$$
\sum_{i}^{\text {occupied }} \varphi_{i}(r) \varphi_{i}^{*}\left(r^{\prime}\right)+\sum_{i}^{\text {virtual }} \varphi_{i}(r) \varphi_{i}^{*}\left(r^{\prime}\right)=\delta\left(r-r^{\prime}\right),
$$

the sum over the virtual orbitals can be eliminated in favor of that on the occupied ones to yield

$$
o_{s}\left(r, r^{\prime}\right)=\delta\left(r-r^{\prime}\right)-p\left(r, r^{\prime}\right),
$$

where $p\left(r, r^{\prime}\right)$ is a projection operator onto the space of the occupied orbitals. For a closed-shell system, $p\left(r, r^{\prime}\right)$ is

$$
p\left(r, r^{\prime}\right)=\sum_{i=1}^{N / 2} \varphi_{i}(r) \varphi_{i}^{*}\left(r^{\prime}\right) .
$$

Two limiting values of $G$ are of special interest: $G=0$ (which transforms $E^{G}$ into the expectation value of the true Hamiltonian) and $G \rightarrow \infty$. As $\int o_{s} \gamma$ is nonnegative, at large $G$, the minimal $\left\langle\hat{H}^{G}\right\rangle$ will be reached when $\int o_{s} \gamma \rightarrow 0$, i.e., when only the occupied (Hartree-Fock or Kohn-Sham) orbitals are used to construct the minimizing wave function. Thus, $E^{G \rightarrow \infty}$ becomes the expectation value of the minimizing wave function in the space present in $p\left(r, r^{\prime}\right)$. With the choice made in Eq. (4), one can now obtain the (Wigner-Löwdin or

${ }^{\ddagger}$ More general forms can be used, e.g., by making a selection within the space of virtual orbitals, yielding room for multideterminant reference wave functions or by having different weights for each of the orbitals, shifting the orbitals in a nonuniform fashion. 
Kohn-Sham) correlation energy (in the "quantum chemistry" or one of the "density functional" definitions, respectively):

$$
E_{c}=E^{G=0}-E^{G \rightarrow \infty} .
$$

A generalized form is

$$
E_{c}^{G}=E^{G}-E^{G \rightarrow \infty} .
$$

According to Eq. (3), $E_{c}^{G}$ can be rewritten as

$$
E_{c}^{G}=-\int_{G}^{\infty} d G \int o_{s}\left(r, r^{\prime}\right) \gamma^{G}\left(r^{\prime}, r\right) d^{3} r d^{3} r^{\prime} .
$$

Please note that this is an explicit formula for the correlation energy in terms of first-order density matrices $\left[\gamma^{G}\left(r^{\prime}, r\right)\right]$ (cf. $[13,14]$ for another possibility). More insight can be gained by expanding $\gamma^{G}$ in terms of the orthonormal orbitals $\phi$ :

$$
\gamma^{G}\left(r^{\prime}, r\right)=\sum_{k, l} P_{k l}^{G} \phi_{k}\left(r^{\prime}\right) \phi_{l}^{*}(r)
$$

( $P_{k l}^{G}$ are expansion coefficients). Then, from Eqs. (2), (6), (8), and (10),

$$
\partial E_{c}^{G} / \partial G=N-\sum_{i=1}^{N / 2} \sum_{k, l}\left\langle\varphi_{i} \mid \phi_{k}\right\rangle P_{k l}^{G}\left\langle\phi_{l} \mid \varphi_{i}\right\rangle,
$$

where $N$ is the number of electrons in the system ( $\gamma$ has been chosen normalized to $N$ ). If the set $\phi$ is chosen to be that of the eigenfunctions at $G \rightarrow \infty$, then

$$
\begin{aligned}
\partial E_{c}^{G} / \partial G & =N-\sum_{i=1}^{N / 2} P_{i i}^{G} \\
& =\sum_{i>N / 2} P_{i i}^{G},
\end{aligned}
$$

which is the number of electrons transferred by correlation into the $G \rightarrow \infty$ unoccupied levels. By integration, the previous equation yields.

$$
E_{c}^{G}=-\int_{G}^{\infty} d G \sum_{i>N / 2} P_{i i}^{G} .
$$

The equations above can be also derived in the case when the wave function is constrained to yield a given density $\rho$ (cf. [15]). Thus, $E_{c}^{G}$ becomes

$$
\min _{\Psi \rightarrow \rho}\left\langle\Psi\left|\hat{H}^{G}\right| \Psi\right\rangle-\lim _{G \rightarrow \infty} \min _{\Phi \rightarrow \rho}\left\langle\Phi\left|\hat{H}^{G}\right| \Phi\right\rangle .
$$

With the above formula, $E_{c}^{G}$ is (for a given $G$ ) a universal functional of the density: The contribution of the terms containing the external potential vanishes, and the projection operator appearing in $\hat{H}^{G}$ can be obtained from the density alone when Kohn-Sham orbitals are used (which, of course, can be obtained from the density alone). In particular, for $G=0$, the correlation energy is (as expected) a universal functional of the density.

We will close this section with a short comment about the significance of the operator $o_{s}$ : With the choice made in Eq. (6), some of the matrix elements of the Hamiltonian $\hat{H}^{G}$ will differ from those of $\hat{H}$. Deriving effective one-body Hamiltonians from $\hat{H}^{G}$ (such as Kohn-Sham or Hartree-Fock) will normally introduce a supplementary dependence on $G$, namely, an upward shift by $G$ of all the unoccupied one-electron levels of the effective one-electron Hamiltonian, increasing the gap between the occupied and unoccupied levels by $G$.

\section{Perturbation Theory}

For large values of $G$, perturbation theory can be applied to second order and yield

$$
E^{G}=E^{G \rightarrow \infty}-C / G+O\left(G^{-2}\right)
$$

where $C$ is a positive constant. To see this, consider the Epstein-Nesbet unperturbed Hamiltonian in the Rayleigh-Schrödinger perturbation theory [16-18]:

$$
\hat{H}_{0}^{G}=\sum_{I}\left|\Phi_{I}\right\rangle\left\langle\Phi_{I}\left|\hat{H}^{G}\right| \Phi_{I}\right\rangle\left\langle\Phi_{I}\right|
$$

$\Phi_{I}$ being Slater determinants. The perturbation operator $\hat{H}^{G}-\hat{H}_{0}^{G}$ has only nondiagonal nonzero elements. There are, thus, no diagonal elements in the numerators of the perturbation energy expansion. Furthermore, the one-body operator $\hat{o}_{s}$ has only nonzero elements on the diagonal which yield with Eq. (6) $\left\langle\Phi_{I}\left|\hat{H}^{G}\right| \Phi_{J}\right\rangle=\left\langle\Phi_{I}|\hat{H}| \Phi_{J}\right\rangle+m_{I} G \delta_{I J}$, where $m_{I}$ is the number of spin-orbitals of $\Phi_{I}$ not present in $p$. Thus, $G$ is present in the denominators only. The first-order contribution to $E^{G}$ vanishes, and the $n>1$ contribution to it will have terms proportional to $1 / G^{n}$ for large $G$. For exam- 
ple, the second-order term

$$
E_{c}^{G, 2}=-\sum_{I \neq 0} \frac{\left\langle\Phi_{0}|\hat{H}| \Phi_{I}\right\rangle\left\langle\Phi_{I}|\hat{H}| \Phi_{0}\right\rangle}{\left\langle\Phi_{I}|\hat{H}| \Phi_{I}\right\rangle-\left\langle\Phi_{0}|\hat{H}| \Phi_{0}\right\rangle+m_{I} G}
$$

becomes for large $G$

$$
E_{c}^{G, 2} \rightarrow-\frac{1}{G} \sum_{I \neq 0} \frac{\left\langle\Phi_{0}|\hat{H}| \Phi_{I}\right\rangle\left\langle\Phi_{I}|\hat{H}| \Phi_{0}\right\rangle}{m_{I}} .
$$

When no singly excited Slater determinants contribute to the $\left\langle\Phi_{I}|\hat{H}| \Phi_{0}\right\rangle$ matrix elements (e.g., when $p$ was constructed from Hartree-Fock orbitals), the expression of $E_{c}^{G, 2}$ can be further simplified:

$$
C=\frac{1}{2} \sum_{I \neq 0}\left\langle\Phi_{0}|\hat{H}| \Phi_{I}\right\rangle\left\langle\Phi_{I}|\hat{H}| \Phi_{0}\right\rangle,
$$

which can also be written as a functional of $\Phi_{0}$ by using $\sum_{I}\left|\Phi_{I}\right\rangle\left\langle\Phi_{I}\right|=1$ :

$$
C=\frac{1}{2}\left[\left\langle\Phi_{0}\left|\hat{H}^{2}\right| \Phi_{0}\right\rangle-\left\langle\Phi_{0}|\hat{H}| \Phi_{0}\right\rangle^{2}\right] .
$$

A consequence of Eq. (14) is that

$$
\frac{\partial E_{c}^{G}}{\partial G}=-\frac{C}{G^{2}}+O\left(G^{-3}\right)
$$

for $G \rightarrow \infty$. As by the definition in Eq. (8), $E_{c}{ }^{G} \rightarrow 0$ for $G \rightarrow \infty$, it follows that for large $G$

$$
E_{c}^{G}=\frac{C}{G}+O\left(G^{-2}\right) .
$$

\section{Approximations}

Is a local approximation better if a uniform electron gas with a gap is used as a reference system? In other words, is it possible to approximate the correlation energy by using the correlation energy per particle of the uniform electron gas with gap $G, \varepsilon_{c, G}(\rho)$ :

$$
E_{c} \approx \int \rho(r) \varepsilon_{c, G}(\rho(r)) d^{3} r .
$$

A short discussion of this first approximation will be made below by using some atoms and ions as a reference. A more general form would be, of course, to choose the gap locally, e.g., by defining a local density of states:

$$
E_{c} \approx \int \rho(r) \varepsilon_{c, G(r)}(\rho(r)) d^{3} r .
$$

One advantage of such a scheme is that extensivity can be guaranteed. ${ }^{\S}$

Another type of a local approximation to $E_{c}$ can be generated from $\partial E_{c}^{G} / \partial G$. By performing just the integration over $r^{\prime}$ in Eq. (11) or by simply using the $\int\left|\varphi_{i}(r)\right|^{2} d^{3} r=1$ in Eq. (12),

$$
\partial E_{c}^{G} / \partial G=\sum_{i>N / 2} P_{i i}^{G} \int\left|\varphi_{i}(r)\right|^{2} d^{3} r,
$$

and introducing the functions $\varepsilon_{c, G}^{\prime}(r)$,

$$
\varepsilon_{c, G}^{\prime}(r)=\frac{\sum_{i>N / 2} P_{i i}^{G}\left|\varphi_{i}(r)\right|^{2}}{\rho(r)}
$$

and

$$
\varepsilon_{c, G}(r)=-\int_{G}^{\infty} \varepsilon_{c, G}^{\prime}(r) d G
$$

Thus,

$$
\partial E_{c}^{G} / \partial G=\int \rho(r) \varepsilon_{c, G}^{\prime}(r) d^{3} r
$$

and

$$
E_{c}^{G}=\int \rho(r) \varepsilon_{c, G}(r) d^{3} r .
$$

We will use the notation $\varepsilon_{c, G=0}=\varepsilon_{c}$ when building $E_{c}$.

As the formulas above are also valid for the uniform electron gas, we can generate a first approximation by assuming that $\varepsilon_{c, G}(r)$, or directly $\varepsilon_{c}(r)$, can be transferred from the uniform electron gas with density $\rho(r)$. This is, of course, nothing but the usual local density approximation (LDA). As $C$ is a monotonous function of the density in the uniform electron gas (it is proportional to $r_{s}^{-2}$, where $\rho=4 \pi r_{s}^{3} / 3$; see below), we can obtain a second approximation by transferring $\varepsilon_{c, G}^{\prime}(r)$ from the the uniform electron gas having the same $C$ (given by $\lim _{G \rightarrow \infty} G^{2} \varepsilon_{c, g}^{\prime}$ ). It is interesting to note that this approximation becomes exact in one-electron systems. There, $C=0$ (any shift of the virtual levels does not change the energy). For the uniform elec-

${ }^{\S}$ A nontrivial global value of $G$ cannot be found in a way to ensure that the energy of the system formed by two different noninteracting subsystems A and B is the same as the sum of the energies of the subsystems: If the systems are different and $G_{A} \neq G_{B}$, which of the $G$ has to be used for the composite system? 
tron gas, $C \rightarrow \infty$ as $r_{s} \rightarrow \infty$; in this limit, the correlation energy per particle vanishes. In other words, transferring the correlation energy per particle from the uniform electron gas with vanishing $C$ means that no correlation energy is transferred, in contrast to the LDA, where a spurious self-interaction is present.

\section{Numerical Results: The Uniform Electron Gas}

To obtain uniform electron gas data, we repeated Freeman's coupled-cluster calculations [19] modified only by adding the operator Gô for vari- ous values of $G$ and $r_{s}$. The results are given in Table I. For applications, it is useful to possess an analytic fit. We used for that purpose a rational function

$$
\varepsilon_{c, G} \approx \frac{\varepsilon_{c}+c_{1} G}{1+c_{2} G+c_{3} G^{2}}
$$

and the following input:

- $\varepsilon_{c}$, the correlation energy per particle at $G=$ 0 ("exact" values [20]).

- $\varepsilon_{c}^{\prime}=\varepsilon_{c, G=0}^{\prime}$, the first derivative of the correlation energy with respect to the gap $G$ at

TABLE 1

Correlation energies per particle, in mHartree, from coupled cluster calculations for different densities [given as $r_{s}=(3 / 4 \pi \rho)^{1 / 3}$ ] and for different gaps $\tilde{G}=G / k_{F}^{2}$, in atomic units; $k_{F}=\left(3 \pi^{2} \rho\right)^{1 / 3}$.

\begin{tabular}{|c|c|c|c|c|c|c|c|c|c|c|}
\hline \multicolumn{11}{|c|}{$r_{s}$} \\
\hline$\tilde{G}$ & 0.1 & 0.2 & 0.3 & 0.4 & 0.5 & 0.6 & 0.7 & 0.8 & 0.9 & \\
\hline 0.0 & -120.8 & -100.9 & -89.6 & -81.9 & -76.1 & -71.5 & -67.6 & -64.4 & -61.6 & \\
\hline 0.1 & -89.8 & -79.3 & -72.6 & -67.6 & -63.8 & -60.6 & -57.9 & -55.5 & -53.5 & \\
\hline 0.2 & -73.3 & -66.5 & -61.9 & -58.3 & -55.5 & -53.1 & -51.0 & -49.2 & -47.6 & \\
\hline 0.3 & -62.5 & -57.7 & -54.3 & -51.6 & -49.4 & -47.5 & -45.8 & -44.4 & -43.1 & \\
\hline 0.4 & -54.8 & -51.2 & -48.5 & -46.4 & -44.6 & -43.1 & -41.7 & -40.5 & -39.4 & \\
\hline 0.5 & -49.0 & -46.1 & -44.0 & -42.2 & -40.7 & -39.5 & -38.3 & -37.3 & -36.4 & \\
\hline 0.6 & -44.4 & -42.0 & -40.3 & -38.8 & -37.6 & -36.5 & -35.5 & -34.6 & -33.8 & \\
\hline 0.7 & -40.6 & -38.7 & -37.2 & -36.0 & -34.9 & -34.0 & -33.1 & -32.4 & -31.7 & \\
\hline 0.8 & -37.5 & -35.9 & -34.6 & -33.5 & -32.6 & -31.8 & -31.1 & -30.4 & -29.8 & \\
\hline 0.9 & -34.9 & -33.5 & -32.4 & -31.4 & -30.6 & -29.9 & -29.2 & -28.7 & -28.1 & \\
\hline 1.0 & -32.6 & -31.4 & -30.4 & -29.6 & -28.9 & -28.2 & -27.7 & -27.1 & -26.6 & \\
\hline 2.0 & -20.1 & -19.6 & -19.3 & -18.9 & -18.6 & -18.4 & -18.1 & -17.9 & -17.5 & \\
\hline 3.0 & -14.7 & -14.5 & -14.3 & -14.1 & -13.9 & -13.8 & -13.6 & -13.5 & -13.4 & \\
\hline 4.0 & -11.6 & -11.5 & -11.4 & -11.3 & -11.2 & -11.1 & -11.0 & -10.9 & -10.8 & \\
\hline 5.0 & -9.7 & -9.6 & -9.5 & -9.4 & -9.3 & -9.3 & -9.2 & -9.1 & -9.1 & \\
\hline 10.0 & -5.3 & -5.3 & -5.2 & -5.2 & -5.2 & -5.2 & -5.1 & -5.1 & -5.1 & \\
\hline \multicolumn{11}{|c|}{$r_{s}$} \\
\hline$\tilde{G}$ & 1 & 2 & 3 & 4 & 5 & 6 & 7 & 8 & 9 & 10 \\
\hline 0.0 & -59.1 & -44.2 & -36.7 & -31.8 & -28.4 & -25.8 & -23.7 & -22.1 & -20.7 & -19.4 \\
\hline 0.1 & -51.6 & -40.0 & -33.7 & -29.6 & -26.6 & -24.3 & -22.5 & -21.0 & -19.7 & -18.6 \\
\hline 0.2 & -46.1 & -36.7 & -31.3 & -27.8 & -25.1 & -23.1 & -21.4 & -20.0 & -18.9 & -17.9 \\
\hline 0.3 & -14.9 & -33.9 & -29.3 & -26.1 & -23.8 & -21.9 & -20.4 & -19.2 & -18.1 & -17.2 \\
\hline 0.4 & -38.4 & -31.6 & -27.6 & -24.7 & -22.6 & -20.9 & -19.5 & -18.4 & -17.4 & -16.5 \\
\hline 0.5 & -35.5 & -29.6 & -26.0 & -23.5 & -21.5 & -20.0 & -18.7 & -17.7 & -16.7 & -15.9 \\
\hline 0.6 & -33.1 & -27.9 & -24.7 & -22.4 & -20.6 & -19.2 & -18.0 & -17.0 & -16.2 & -15.4 \\
\hline 0.7 & -31.0 & -26.4 & -23.5 & -21.4 & -19.8 & -18.4 & -17.3 & -16.4 & -15.6 & -14.9 \\
\hline 0.8 & -29.2 & -25.1 & -22.4 & -20.5 & -19.0 & -17.8 & -16.7 & -15.9 & -15.1 & -14.4 \\
\hline 0.9 & -27.6 & -23.9 & -21.4 & -19.7 & -18.3 & -17.1 & -16.2 & -15.4 & -14.6 & -14.0 \\
\hline 1.0 & -26.2 & -22.8 & -20.6 & -18.9 & -17.6 & -16.5 & -15.6 & -14.9 & -14.2 & -13.6 \\
\hline 2.0 & -17.5 & -15.9 & -14.7 & -13.8 & -13.1 & -12.5 & -11.9 & -11.5 & -11.0 & -10.7 \\
\hline 3.0 & -13.2 & -12.3 & -11.6 & -11.0 & -10.5 & -10.1 & -9.7 & -9.4 & -9.1 & -8.8 \\
\hline 4.0 & -10.7 & -10.1 & -9.6 & -9.2 & -8.8 & -8.5 & -8.2 & -8.0 & -7.8 & -7.6 \\
\hline 5.0 & -9.0 & -8.5 & -8.2 & -7.9 & -7.6 & -7.4 & -7.2 & -7.0 & -6.8 & -6.6 \\
\hline 10.0 & -5.1 & -4.9 & -4.8 & -4.7 & -4.6 & -4.5 & -4.4 & -4.3 & -4.2 & -4.2 \\
\hline
\end{tabular}


$G=0$ (finite difference coupled cluster value; cf. Table II).

- $\varepsilon_{c}^{\prime \prime}=\varepsilon_{c, G=0}^{\prime \prime}$, the second derivative of the correlation energy $\varepsilon_{c, G}^{\prime \prime}$ with respect to the gap $G$ at $G=0$ (finite difference coupled cluster value; cf. Table II).

- The coefficient $-C$ of $1 / G$ at $G \rightarrow \infty$ (extrapolation of the coupled cluster or secondorder perturbation theory values of $G \varepsilon_{c}$ ).

More explicitly,

$$
\begin{aligned}
\varepsilon_{c}^{\prime} & =c_{1}-\varepsilon_{c} c_{2} \\
\varepsilon_{c}^{\prime \prime} & =2\left[\varepsilon_{c}\left(c_{2}^{2}-c_{3}\right)-c_{1} c_{2}\right] \\
C & =-c_{1} / c_{3}
\end{aligned}
$$

or

$$
\begin{aligned}
& c_{1}=C \frac{2\left(\varepsilon_{c}^{\prime}\right)^{2}-\varepsilon_{c} \varepsilon_{c}^{\prime \prime}}{2\left(C \varepsilon_{c}^{\prime}-\varepsilon_{c}^{2}\right)} \\
& c_{2}=\frac{2 \varepsilon_{c} \varepsilon_{c}^{\prime}-C \varepsilon_{c}^{\prime \prime}}{2\left(C \varepsilon_{c}^{\prime}-\epsilon_{c}^{2}\right)} \\
& c_{3}=-\frac{2\left(\varepsilon_{c}^{\prime}\right)^{2}-\varepsilon_{c} \varepsilon_{c}^{\prime \prime}}{2\left(C \varepsilon_{c}^{\prime}-\varepsilon_{c}^{2}\right)} .
\end{aligned}
$$

TABLE II

First and second derivative of the correlation energies at the gap $G=0$, from coupled cluster calculations, for different densities (given as $\left.r_{s}=(3 / 4 \pi \rho)^{1 / 3}\right)$, in atomic units.

\begin{tabular}{rcl}
\hline$r_{s}$ & $\varepsilon_{c}^{\prime}$ & \multicolumn{1}{c}{$\varepsilon_{c}^{\prime \prime}$} \\
\hline 0.1 & $1.1349 \cdot 10^{-3}$ & $-2.3902 \cdot 10^{-5}$ \\
0.2 & $2.9533 \cdot 10^{-3}$ & $-1.9218 \cdot 10^{-4}$ \\
0.3 & $5.0630 \cdot 10^{-3}$ & $-6.3269 \cdot 10^{-4}$ \\
0.4 & $7.3488 \cdot 10^{-3}$ & $-1.4544 \cdot 10^{-3}$ \\
0.5 & $9.7524 \cdot 10^{-3}$ & $-2.7522 \cdot 10^{-3}$ \\
0.6 & $1.2239 \cdot 10^{-2}$ & $-4.6096 \cdot 10^{-3}$ \\
0.7 & $1.4786 \cdot 10^{-2}$ & $-7.1022 \cdot 10^{-3}$ \\
0.8 & $1.7376 \cdot 10^{-2}$ & $-1.0296 \cdot 10^{-2}$ \\
0.9 & $1.9930 \cdot 10^{-2}$ & $-1.4081 \cdot 10^{-2}$ \\
1.0 & $2.2502 \cdot 10^{-2}$ & $-1.8763 \cdot 10^{-2}$ \\
2.0 & $4.9413 \cdot 10^{-2}$ & $-1.2117 \cdot 10^{-1}$ \\
3.0 & $7.5618 \cdot 10^{-2}$ & $-3.4170 \cdot 10^{-1}$ \\
4.0 & $1.0118 \cdot 10^{-1}$ & $-7.0947 \cdot 10^{-1}$ \\
5.0 & $1.2577 \cdot 10^{-1}$ & -1.2313 \\
6.0 & $1.4959 \cdot 10^{-1}$ & -1.9209 \\
7.0 & $1.7268 \cdot 10^{-1}$ & -2.7861 \\
8.0 & $1.9515 \cdot 10^{-1}$ & -3.8347 \\
9.0 & $2.1714 \cdot 10^{-1}$ & -5.0776 \\
10.0 & $2.3508 \cdot 10^{-1}$ & -6.2832 \\
\hline
\end{tabular}

The density-dependence of $\varepsilon_{c}^{\prime}$ was assumed to be well represented by

$$
\varepsilon_{c}^{\prime} \approx \frac{a_{1} r_{s}^{3 / 2}}{1+a_{2} r_{s}^{1 / 2}+a_{3} r_{s}+a_{1} r_{s}^{3 / 2}},
$$

which has the correct type of behavior at low $r_{s}$ [8]. For $r_{s} \rightarrow \infty$, we used Eq. (25) and the Wigner crystal momentum distribution of March and Sampanthar [21,22] which give a Gaussian momentum distribution, the exponent vanishing with $r_{s} \rightarrow \infty$, meaning that in this limit a negligible fraction of the electrons remain in the occupied orbitals. The values obtained for the coefficients are $a_{1}=0.04953, a_{2}=1.07024$, and $a_{3}=0.07928$. Equation (12) establishes a relationship between $\varepsilon_{c}^{\prime}$ and the relative number of electrons in momentum states $q>k_{F}$. Our values compare favorably with results from the literature [23-25]. Although a more detailed analysis is certainly feasible, we simply assumed that $\varepsilon_{c}^{\prime \prime}$ can be approximated by a polynomial in $r_{s}$. We got a reasonable fit (for $\left.0.1 \leq r_{s} \leq 10\right)$ with

$$
\varepsilon_{c}^{\prime \prime} \approx \sum_{i=3}^{7} b_{i} r_{s}^{i}
$$

where

$$
\begin{aligned}
& b_{3}=-0.02504, \\
& b_{4}=0.007026, \\
& b_{5}=-0.001268, \\
& b_{6}=0.0001136,
\end{aligned}
$$

and

$$
b_{7}=-0.000003841 \text {. }
$$

As $C$ is given by second-order perturbation theory, a simple scaling shows that it should be proportional to $k_{F}^{+2}$. We computed the proportionality coefficient to be equal to 0.06483 Hartree."

The quality of the fit was checked by comparison with the calculated values of $\varepsilon_{c}^{G}$. The errors of the fit are comparable to those of the coupled cluster calculation in the range analyzed."

\footnotetext{
" The "direct" part can be found analytically to be $14 /\left(15 \pi^{2}\right)$ Hartrees.

\# With the exception of the dependence of $\varepsilon_{c}^{\prime}$ for small $r_{s}$ [8], we did not take care of the analytic behavior of the coefficients; their quality may become worse when used beyond the range of density values used in our calculations.
} 


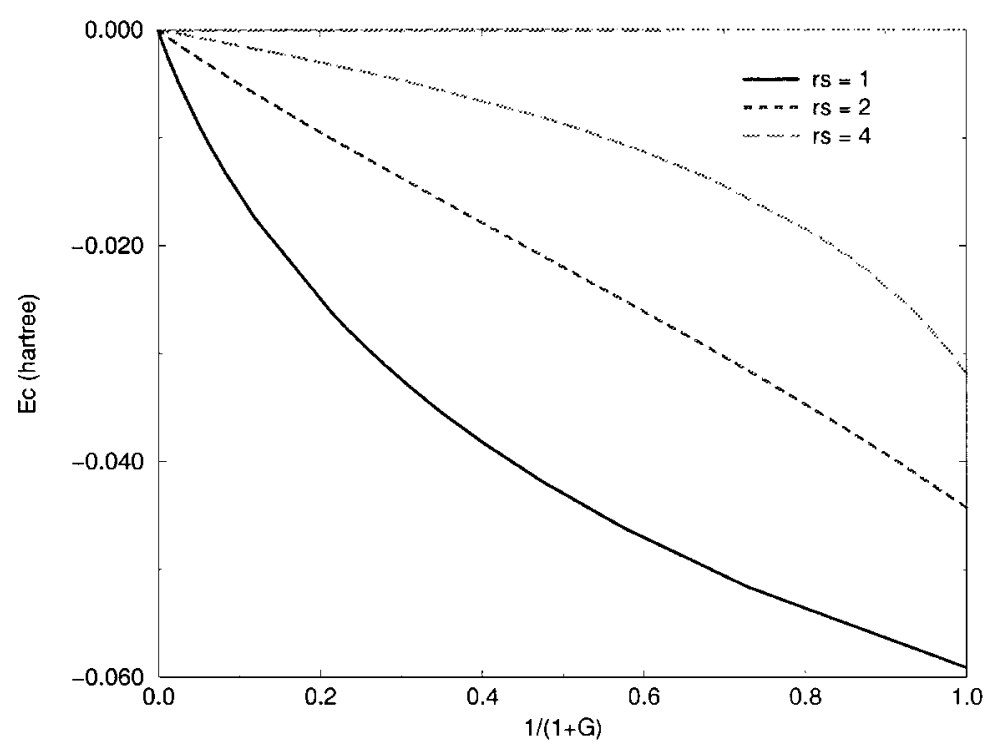

FIGURE 1. Correlation energy per particle in the uniform electron gas with increasing gap [as a function of $1 /(1+G)$ ] ( $r s=1$, full curve; $r s=2$, short-dashed curve; $r s=4$, long-dashed curve).

The behavior of $\varepsilon_{c}$ as a function of the gap is shown in Figure 1 for different values of $r_{s}$. [Please notice that we have chosen to use as the abscissa $1 /(1+G)$, the left-hand end corresponding to the noninteracting system, and the right-hand end, to the real one.) The sign of the curvature changes with the density of the gas: It is positive for high and negative for low densities.

\section{Numerical Results: Some Atoms and Ions}

To obtain some insight into the effect of the approximations, we performed some calculations on small atoms and ions using Gaussian basis sets and the program Molpro [26]. We performed configuration interaction calculations for the $\mathrm{He}$ and the Be series to obtain reliable densities and correlation energies. In Figure 2, we show the change of the correlation energy per particle with $G$ for the $\mathrm{He}$ and the Be atom. While He seems to behave like a high-density uniform electron gas, Be shows a new type of curve. A more detailed analysis shows, however, that this first impression can be corrected: When only the valence shell of the Be atom is correlated, the curve has the curvature similar to that of a low-density uniform electron gas, while the core of the Be atom behaves like a high-density electron gas (cf. Fig. 3).
To determine an order of magnitude for $G$ in the first approximation discussed above, we fixed $G$ by requiring that the approximate correlation energy equals the exact one. The resulting values of $G$ are plotted in Figure 4 vs. the ionization potential of the atom/ion. For the He series, the behavior is quite regular: $G$ is approximately five times larger than the ionization potential. At first, this hugh gap might be surprising. One should remember, however, that the orbitals needed to describe the correlation are different from those describing the excited states (the natural orbitals are in the region of the occupied orbitals, unlike the diffuse Rydberg orbitals [27]. One can expect that the use of a local gap might considerably improve the results, e.g., by considering a measure of the overlap of the occupied and virtual states. We expect that exploring the density of the unoccupied states will help improve the results, as the density of the Rydberg states overlaps only a little with that of the occupied states. In the Be series, $G$ is first increasing, but stabilizes for heavier nuclei. We expect that the continuation of this curve will be decreasing, finally crossing the abscissa: There will be no positive gap allowing the equalization of the correlation energy of the ion with that of a uniform electron gas with a gap. We deduce this behavior from Figure 3 of [28], where one can guess that with increasing nuclear charge the LDA correlation energy will be higher than the real one. 


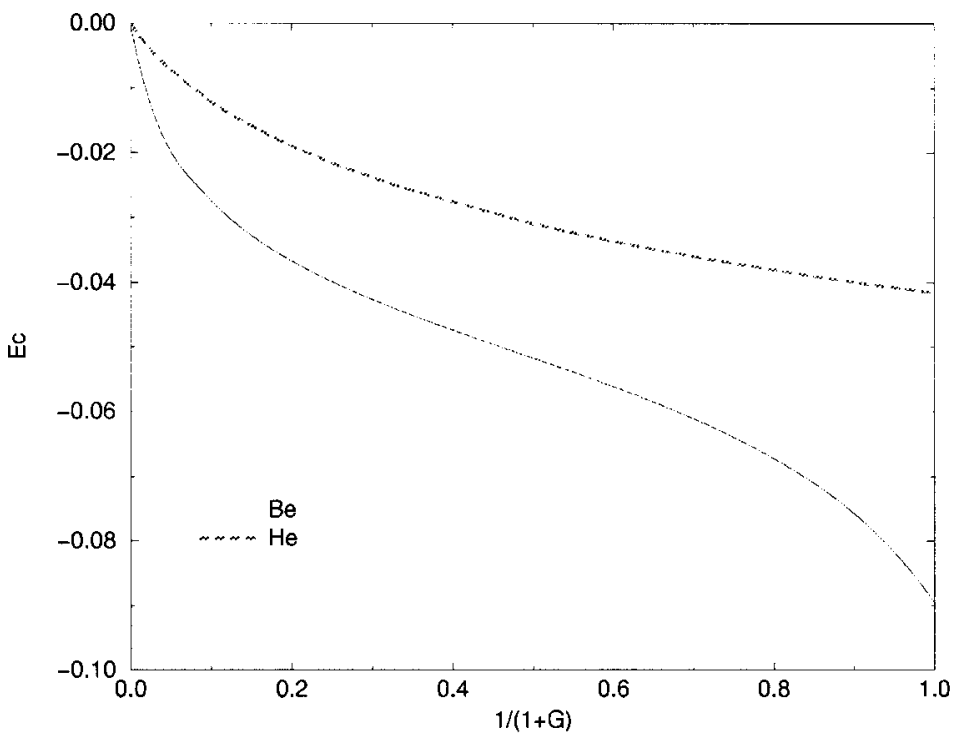

FIGURE 2. Correlation energy for the $\mathrm{He}$ (dashed line) and the Be atom (dotted curve) as functions of $1 /(1+G)$, in atomic units.

As there is no (positive) gap capable of lowering the correlation energy of the uniform electron gas, we deduce the impossibility of correctly representing the correlation energy of such strongly correlated systems with the approximation given above. Of course, one could try to include the orbitals producing the strong correlation $(2 p)$ into the pro- jection operator $p\left(r, r^{\prime}\right)$ and the problem just mentioned will disappear.

Let us now discuss the second approximation (the transfer of $C$ from the uniform electron gas). We used for this exploratory calculation Hartree-Fock orbitals to define the projector $p$ and performed a quantum chemical (multi-

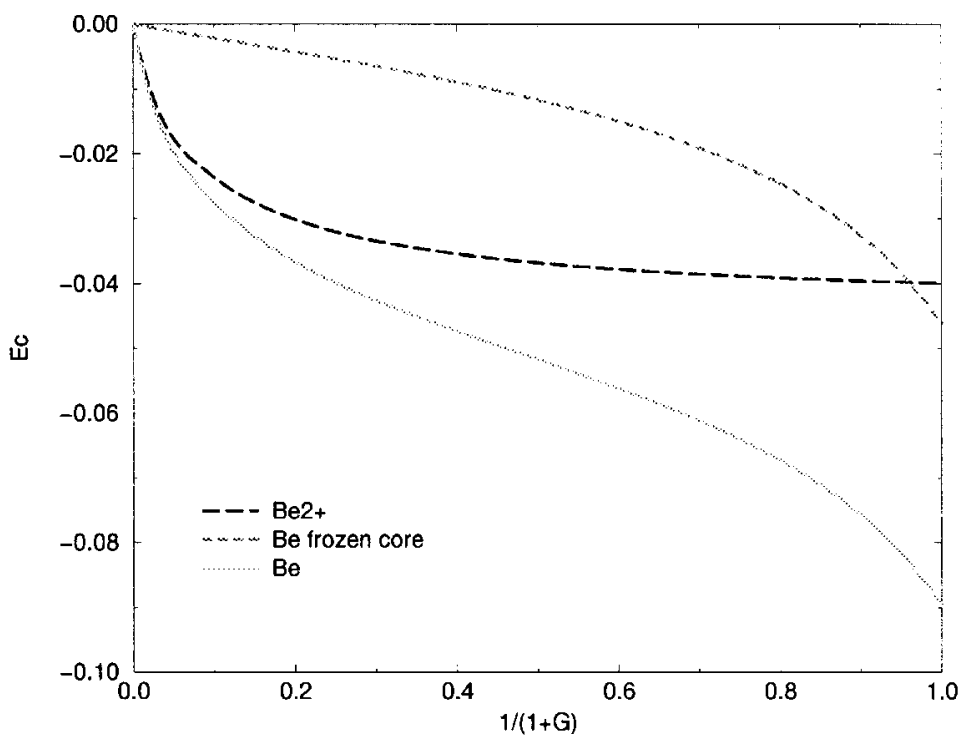

FIGURE 3. Correlation energy for the BE (dotted curve), that obtained by correlating the valence only (frozen core, short-dashed curve) and the Be core (Be ${ }^{2+}$, long-dashed curve), as functions of $1 /(1+G)$, in atomic units. 


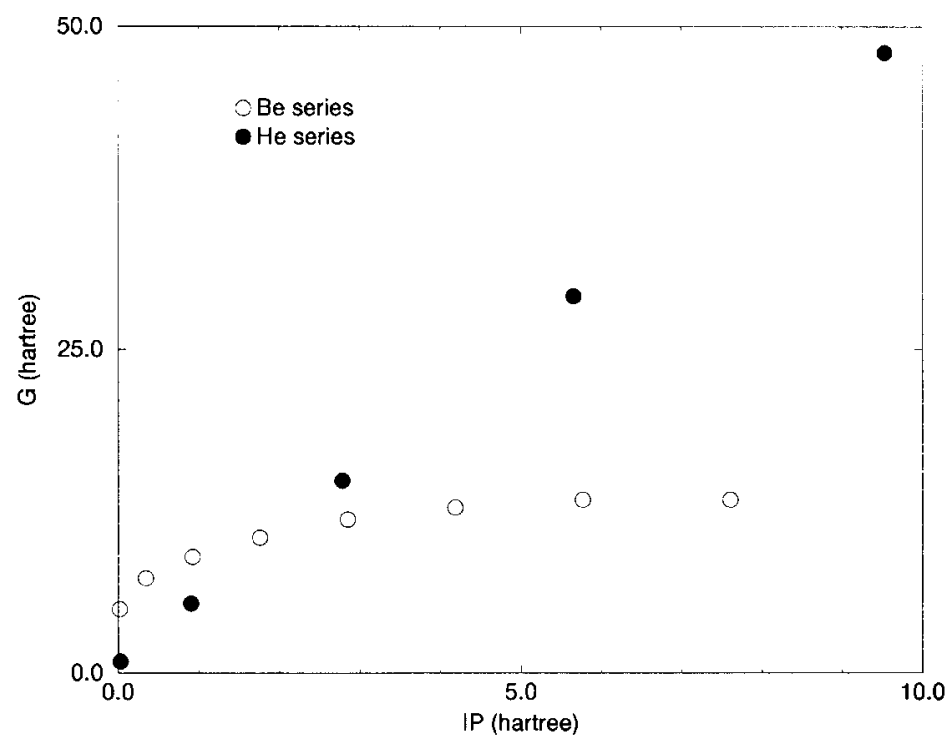

FIGURE 4. Gap yielding the exact correlation energy in the local approximation in the He series (full circles) and in the Be series (empty circles).

configuration) calculation for a large value of $G$ (typically 50 or 100) to obtain in each point of space $\varepsilon_{c, G}^{\prime}$ according to Eq. (25). We next obtained in each point of the space $G^{2} \varepsilon_{c, G}^{\prime}(r)=C(r)$ and numerically integrated

$$
\int d^{3} r \rho(r) \varepsilon_{c}^{u n i f}[C(r)]
$$

where $\varepsilon_{c}^{\text {unif }}[C(r)]$ is the correlation energy per particle for the uniform electron gas having the asymptotic behavior $-C(r) / G^{* *}$ We obtained for the correlation energy of the He Atom 0.09 Hartree (which is in between the exact value of 0.04 and that obtained with LDA at $G=0,0.11$ Hartree). Similarly, we obtain for the Be atom 0.17 Hartree ("exact" 0.09; LDA at $G=0$ : 0.22).

The previous approximation can be refined by considering a nonzero gap. By simply choosing the gap equal to the ionization potential (or the value of the eigenvalue of the last occupied Kohn-Sham orbital: 0.90 for $\mathrm{He}$ and 0.34 for $\mathrm{Be}$ ), we obtain a considerable improvement: 0.05 Hartree for $\mathrm{He}$ and 0.13 Hartree for Be. (The local density approximation values obtained by choosing the gap equal to the ionization potential are 0.08 and 0.19 Hartree, for $\mathrm{He}$ and $\mathrm{Be}$, respectively.)

\footnotetext{
** In the special case of the He atom, as only one orbital is occupied at $G \rightarrow \infty$, and the density is chosen as the Hartree-Fock one; $C(r)$ is a constant which turns to be that of a uniform electron gas with $r_{s} \approx 2$.
}

\section{Conclusion}

We hope that using model Hamiltonians with an additional nonlocal one-body operator may help one to understand density functional theory and to devise new methods. A new adiabatic coupling between the real system and the noninteracting one (with all unoccupied levels shifted up to infinity) can be used to define the correlation energy. The formula obtained shows a relationship between the latter and an electron transfer from the noninteracting occupied orbitals to the unoccupied ones, due to correlation. Perturbation theory can be used to construct the first correction for very large, but finite gaps. Under certain conditions, the present perturbation theory yields terms depending on the reference determinant only, the price to be paid being expectation values of powers of the true Hamiltonian $H$.

Uniform electron gas calculations have been performed for different densities and values of the gap parameter G. An analytical approximation is given for the correlation energy, as well as for its first and second derivative with respect to $G$ at zero gap.

Numerical results show that a local approximation produced by transferring the correlation energy per particle from a uniform electron gas with 
a gap to simple atomic systems requires values for the gap which are larger than the atomic ionization potential. An alternative method was also presented, in which the perturbation theory secondorder term is correctly treated and extrapolated to a zero gap by using density functional data. This approach automatically is self-interaction free. Numerical results indicate, however, that a more sophisticated treatment is required to produce correlation energies with the quality required nowadays.

\section{ACKNOWLEDGMENTS}

We are grateful to F. Colonna, J. Dobson, W. Kohn, W. Pickett, C. Umrigar, and U. von Barth for stimulating discussions, to $\mathrm{H}$. J. Werner for the $\mathrm{ab}$ initio program MOLPRO, and to J. Krieger and M. Levy for comments on the manuscript.

\section{References}

1. D. C. Langreth and J. P. Perdew, Solid State Commun. 17, 1425 (1975).

2. O. Gunnarsson and B. Lundqvist, Phys. Rev. B 13, 4274 (1976).

3. A. D. Becke, J. Chem. Phys. 98, 1372 (1993).

4. Q. Zhao, M. Levy, and R. G. Parr, Phys. Rev. A 47, 918 (1993).

5. A. Savin and H.-J. Flad, Int. J. Quantum Chem. 56, 327 (1995).
6. G. Engel and W. E. Picket, Phys. Rev. B 54, 8420 (1996).

7. W. Kohn and L. Sham, Phys. Rev. A 140, 1133 (1965).

8. J. Callaway, Phys. Rev. 116, 1368 (1959).

9. D. Penn, Phys. Rev. 128, 2093 (1962).

10. Z. H. Levine and S. Louie, Phys. Rev. B 25, 6310 (1982).

11. W. E. Pickett and C. S. Wang, Phys. Rev. B 30, 4719 (1984).

12. H. Nakatsuji and R. G. Parr, J. Chem. Phys. 63, 1112 (1975).

13. A. Savin, Phys. Rev. A 52, R1805 (1995).

14. M. Levy and A. Görling, Phys. Rev. A 52, R1808 (1995).

15. M. Levy, Proc. Natl. Acad. Sci. U.S.A. 76, 6062 (1979).

16. P. S. Epstein, Phys. Rev. 28, 695 (1926).

17. R. K. Nesbet, Proc. R. Soc. Lond. A 230, 312 (1955).

18. R. Nesbet, Proc. R. Soc. Lond. A 230, 322 (1955).

19. D. L. Freeman, Phys. Rev. B 15, 5512 (1977).

20. S. Vosko, L. Wilk, and M. Nusair, Can. J. Phys. 58, 1200 (1980).

21. N. H. March and S. Sampanthar, Acta Phys. Hung. 61, 61 (1962).

22. N. March, W. Young, and S. Sampanthar, The Many-Body Problem in Quantum Mechanics (Cambridge University Press, Cambridge, 1967).

23. E. Pajanne and J. Arponen, J. Phys. C: Solid State Phys. 15, 2683 (1982).

24. Y. Takada and H. Yasuhara, Phys. Rev. B 44, 7879 (1991).

25. G. Ortiz and P. Ballone, Phys. Rev. B 50, 1391 (1994).

26. MOLPRO is a package of ab initio programs written by H.-J. Werner and P. J. Knowles, with contributions from J. Almlöf, R. D. Amos, A. Berning, M. J. O. Deegan, F. Eckert, S. T. Elbert, C. Hampel, R. Lindh, W. Meyer, A. Nicklass, K. Peterson, R. Pitzer, A. J. Stone, P. R. Taylor, M. E. Mura, P. Pulay, M. Schuetz, H. Stoll, T. Thorsteinsson, and D. L. Cooper.

27. E. R. Davidson, Rev. Mod. Phys. 44, 451 (1972).

28. J. Perdew, E. R. McMullen, and A. Zunger, Phys. Rev. A 23, 2785 (1981). 